\title{
Differences in Early Season Emergence and Reproductive Activity Between Spathius agrili (Hymenoptera: Braconidae) and Spathius galinae, Larval Parasitoids of the Invasive Emerald Ash Borer (Coleoptera: Buprestidae)
}

\author{
Max Ragozzino, ${ }^{1,4, \oplus}$ Ryan Meyer, ${ }^{1}$ Jian Duan, ${ }^{2}$ Ben Slager, ${ }^{3}$ and Scott Salom ${ }^{1}$ \\ 'Department of Entomology, Virginia Tech, Price Hall, Blacksburg, VA 24061, ${ }^{2}$ USDA ARS BIIRU 501 S. Chapel St, Newark, DE 19702, \\ ${ }^{3}$ USDA APHIS PPQ 5936 Ford Court Suite 200, Brighton, MI 48116, and ${ }^{4}$ Corresponding author, e-mail: maxri@vt.edu \\ Subject Editor: Rebecca Schmidt-Jeffris \\ Received 13 August 2019; Editorial decision 20 December 2019
}

\begin{abstract}
Both Spathius agrili Yang and Spathius galinae Belokobylskij and Strazanac are host-specific parasitic wasps introduced for biological control of emerald ash borer in North America. Spathius agrili is native to northeastern China and S. galinae comes from a more northern, colder climate in the Russian Far East. Their origin may lead to differing abilities to adapt to climate and their host in North America. We conducted both field and laboratory experiments to determine the timing of early season emergence and synchronization of each parasitoid species to their host in the United States, and if manipulating prerelease conditions could affect emergence time. A cold acclimatization treatment prior to parasitoid emergence was assessed and compared with untreated control group reared with standard rearing protocols. Stands of naturally emerald ash borer-infested ash were sampled at two locations in Virginia throughout the experiment to determine when the parasitoid-susceptible life stage (third to fourth instar) occurred. Untreated S. galinae emerged approximately 2 wk earlier than any other cohort, whereas cold acclimatized $S$. galinae emerged later than any other cohort. Emergence time of $S$. agrili was unaffected by cold acclimatization. Cold acclimatization treatment did not affect the parasitism rate of either species, nor did it have multigenerational effects. Emergence time of the subsequent generation of $S$. agrili was delayed by cold acclimatization treatment, whereas $S$. galinae experienced no multigenerational effects. At Virginia field sites, susceptible EAB larvae were present during the emergence time of all four groups of parasitoids. Untreated S. galinae had the least overlap with any susceptible EAB larvae.
\end{abstract}

Key words: emerald ash borer, Spathius agrili, Spathius galinae, climate

The emerald ash borer (EAB), Agrilus planipennis Fairemaire, a pest of ash (Fraxinus spp.) trees native to Asia, was accidentally introduced to North America in the mid-1990s (Cappaert et al. 2005, Bray et al. 2011, Siegert et al. 2014). Since it was first discovered in 2002 in southeastern Detroit, Michigan and Ontario, Canada (Haack et al. 2002), this invasive beetle has established populations in over 35 U.S. states and five Canadian provinces, killing millions of North American ash trees by 2019 (Emerald Ash Borer Information 2019). Currently, management of this invasive beetle in North America involves several options, including trunk injections of systemic pesticides, removal of infested trees, and biological control with specialized natural enemies (parasitoids) from the pest's native range (Bauer et al. 2008, Mercader et al. 2015). Because of the lack of efficient detection methods and difficulty of applying insecticides in natural forests, biological control is considered to be a critical long-term option for protection of ash stands in natural ecosystems (Duan et al. 2018).

Three hymenopteran parasitoids were introduced from northeast China, part of the pest's native range, in 2007 as biocontrol agents against EAB in the United States (Bauer et al. 2007, 2008; Liu et al. 2007). These included two larval parasitoids, Tetrastichus planipennisi Yang (Hymenoptera: Eulophidae; Yang et al. 2006) and Spathius agrili Yang (Yang et al. 2005), and the egg parasitoid Oobius agrili Zhang and Huang (Hymenoptera: Encyrtidae; Zhang et al. 2005). To date, these three Chinese parasitoids have been released in 29 U.S. states as well as three of the five Canadian provinces invaded by EAB (Duan et al. 2018, Canadian Food Inspection Agency 2019, MapBiocontrol 2019). Releases of S. agrili and T. planipennisi occur either as mature adults, larvae, or pupae in ash bolts to be hung on infested ash trees depending on availability (USDA-APHIS/ARS/ 
FS 2019). Recent field studies conducted in Midwest, mid-Atlantic, and northeastern United States have shown that T. planipennisi and O. agrili have established self-sustaining populations in many of the released areas in these regions (Duan et al. 2013, 2015; Davidson et al. 2016; Jennings et al. 2016). However, none of the published studies have confirmed the establishment of $S$. agrili from these regions (Hooie et al. 2015, MapBiocontrol 2019). Reasons for the failure of $S$. agrili to establish are not exactly known. Some hypotheses include a combination of biotic and abiotic factors, such as the asynchronization of adult parasitoid emergence with EAB larvae, or climatic conditions at release sites. Cold storage of mass reared adults has been attempted, which showed that a cold storage treatment at $10^{\circ} \mathrm{C}$ for $3 \mathrm{mo}$ or longer significantly decreased emergence, longevity, and fecundity of S. agrili (Gould et al. 2011).

Recently, another larval parasitoid, Spathius galinae Belokobylskij and Strazanac was collected in the Russian Far East, north of areas where $S$. agrili was collected in Tianjin province, China (Belokobylskij et. al. 2012, Duan et al. 2012). It was approved for release in the United States in 2015 (Duan et al. 2015, Federal Register 2015, USDA-APHIS/ARS/FS 2019). Pre-release climate matching indicated that $S$. galinae is well suited to the climate of the northcentral and northeastern United States, where ash is abundant (Duan et al. 2018, USDA-APHIS/ARS/FS 2019). Releases of S. galinae also occur by two methods; adult release or larvae/pupae in ash bolts hung on infested ash trees (USDA-APHIS/ARS/FS 2019). In 2016, releases of $S$. galinae were made in several northeastern U.S. states, followed later by releases in the northcentral and Great Lake states (MapBiocontrol 2019). A recent field study conducted in the northeastern United States showed that this newly introduced EAB larval parasitoid successfully established self-sustaining populations with significantly increased abundance or density two years after the major field releases in six hardwood forests in Connecticut, Massachusetts, and New York (Duan et al. 2019). However, little information is available on this parasitoid's ability to reproduce and establish in the mid-Atlantic and southern regions of the United States, where EAB has expanded its range.

The two congener species, S. agrili and S. galinae, share many life history traits that are promising for biological control. Both species are idiobiont larval parasitoids, paralyzing their host upon attack, have increased clutch size with increased host larval size, and show preference for third- and fourth-stage EAB larvae (Yang et al. 2006, 2010; Belokobylskij et al. 2012; Duan et al. 2014; Watt and Duan 2014). Differences between species arise in longevity of adults, fecundity, and sex ratio. Spathius agrili adults lived for an average of 8 wk with males living slightly longer (Gould et al. 2011), whereas S. galinae adults lived for a median $7 \mathrm{wk}$ with females living slightly longer (Duan et al. 2014). Fecundity and sex ratios between these congener species differ as well. Spathius agrili has an average clutch size of $5.4 \pm 0.2$ eggs and is capable of laying 51.2 eggs over her life with approximately three quarters female sex ratio (Gould et al. 2011). Spathius galinae has an between eight and 16 offspring per host and is capable of producing up to an average 47 progeny over her life with a sex ratio (f:m) of 1.9:1 (Belokobylskij et al. 2012, Duan et al. 2014, Watt et al. 2016).

In the present study, we first determine the early-season adult emergence phenology of both these two congener species $S$. agrili and $S$. galinae and assess a $7-\mathrm{d}$ cold acclimatization as a method of delaying their emergence. Secondly, we evaluate if a 7 - $d$ cold acclimatization treatment affects the longevity, parasitism rate, sex ratio, and/or fecundity of cold acclimatized wasps. Finally, we evaluate the subsequent generation, to ensure no multigenerational effects occurred. We hypothesized that a cold acclimatization treatment would alter the emergence time of early season adult parasitoids and affect the seasonal synchronization between parasitoid adults and suitable EAB larvae. We also hypothesized that the cold acclimatization treatment would decrease longevity, parasitism rate, fecundity, and would have a multigenerational effect on reproduction.

\section{Materials and Methods}

\section{Host Larvae for Fecundity and Reproduction Experiment}

All EAB larvae used in the fecundity and reproduction experiments were third or fourth instars reared at the USDA Beneficial Insect Introduction Unit (BIIRU) in Newark, DE. EAB larvae were reared in tropical ash, F. uhdei Wenz., bolts (1-2 cm diam, 20-25 cm length), using the methods described in Duan et al. (2012). Between three and seven larvae were reared per bolt, depending on bolt diameter and length. Larval-inoculated bolts were shipped to the Price's Fork Research Station (PFRC), in Blacksburg, VA in an insulated cooler weekly from May through September. The base of each bolt was wrapped in a paper towel soaked in distilled water, and all bolts were placed in a black plastic bag to prevent desiccation prior to use.

\section{Early Season Emergence}

All $S$. agrili and $S$. galinae cocoons used in this experiment were reared by the USDA APHIS PPQ Biological Control Production Facility, in Brighton, MI (afterward referred to as the "APHIS Brighton Facility"). Green ash, F. pennsylvanica Marshall, bolts $(12-15 \mathrm{~cm}, 2-4 \mathrm{~cm}$ dia.) were infested with EAB that were allowed to mature until the third to fourth instars. Infested bolts were then subjected to gravid females of either $S$. agrili or S. galinae for 7 d. Two treatment groups were established: The control group reared to late-instar larvae using the standard rearing condition of $28^{\circ} \mathrm{C}$ used by the APHIS Brighton Facility, and a group reared at $28^{\circ} \mathrm{C}$ then exposed to a cold acclimatization treatment of $10^{\circ} \mathrm{C}$ for $1 \mathrm{wk}$ as late-instar larvae. Each treatment group was established for both S. agrili and S. galinae, for a total of four cohorts of Spathius spp.: cold acclimatized $S$. agrili, cold acclimatized $S$. galinae, untreated $S$. agrili, and untreated $S$. galinae. Forty bolts of each cohort were produced and then shipped to Virginia Tech.

Each bolt was placed individually in a mesh-ventilated 3.78-liter square plastic container (Plastic Grip Jug 1 Gallon S-15711 ULine Plastics) in a brick of water-soaked floral foam (Oasis). Containers were placed outdoor in the shade under a stand of 20-yr old conifer trees at PFRC $(37.212273,-80.489594)$ on 8 March 2017, and a tarp was hung above them to prevent rain water accumulation in the containers. Temperature was measured constantly using a $\mathrm{HOBO}$ Pro v2 temperature monitor (Fig. 1). Containers were observed twice per week until any emergence was first observed, at which point they were observed daily. All observed wasps were sexed, and a subset were used for further experiments. After emergence ended and no parasitoids emerged for $7 \mathrm{~d}$, bolts were observed twice weekly for any delayed emergence until 1 October 2017.

\section{Early Season Longevity, Parasitism Rate, Sex Ratio, and Fecundity}

After emergence, pairs of male and female wasps were taken from each cohort and placed in a new mesh ventilated container (as described above), containing one to two bolts of tropical ash infested with a total of three to seven third- to fourth-instar EAB. The bottom $1-2 \mathrm{~cm}$ of each bolt were placed in saturated floral foam. Clover honey was streaked on top of the mesh ventilated lid to serve as a 


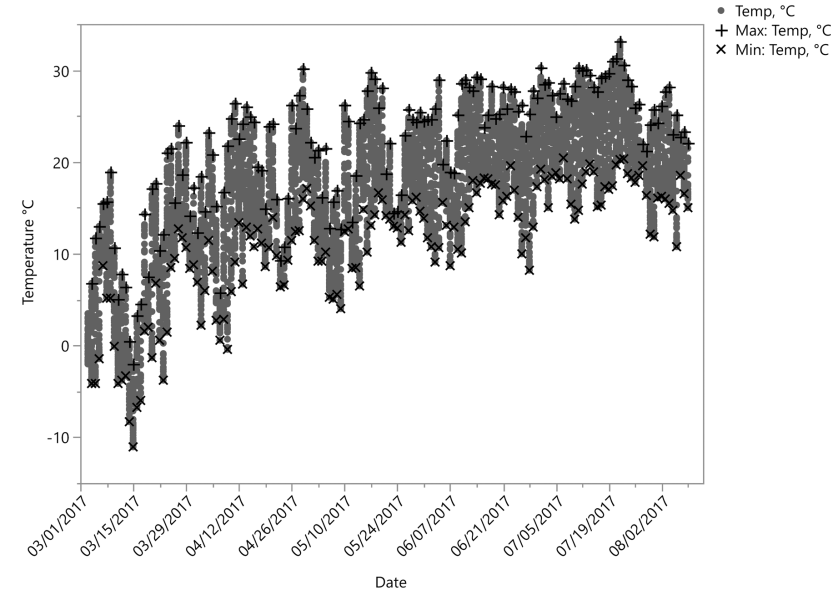

Fig. 1. Daily temperature data from the hemlock stand where bolts were placed at Prices Fork Research Station, Blacksburg, VA. Temperature was recorded every $30 \mathrm{~min}$. Daily high and low temperatures are indicated by + and $x$, respectively.

food source for the adult Spathius. Containers were placed in an incubation growth chamber (I-41LL, Percival Scientific) set to the average weekly day and night temperature of Blacksburg, VA (Fig. 1), matching photoperiod, and $60 \pm 10 \%$ relative humidity in order to mimic field conditions. Containers were observed daily. After $1 \mathrm{wk}$, the mated pair was removed from the container and was placed in a new container with new bolts of EAB-infested tropical ash. This was repeated weekly until the female Spathius died. In the event the male died, the female was moved alone. Longevity of adult parasitoids was determined by observing these mating pairs date of death.

After paired adults were removed from a container, the container was observed daily for progeny emergence, until seven consecutive days of no emergence. Once emergence had completed, bolts were dissected using a sharp utility knife to remove only the outermost layer of bark, revealing the $\mathrm{EAB}$ galleries and parasitoid cocoons. The fate of each EAB larvae was scored as healthy, parasitized (with presence of parasitoid cocoons or larvae), or killed by other (unknown) factors. To determine fecundity, any unemerged parasitoid adults, pupae, and larvae were counted and added to the count of emerged parasitoids.

\section{Subsequent Generation Emergence}

Newly emerged adults were removed from the container on the day they were observed, and sexed. Emergence time was calculated as days since EAB larvae were exposed to paired adults until emergence of the new adult Spathius by individual. This information was tabulated by cohort to determine parasitism rate and time to emergence by cohort.

\section{Subsequent Generation Parasitism Rate}

After adults emerged, they were paired within cohort and placed in a mesh ventilated plastic container containing one to two bolts of EAB-infested tropical ash (as described above). As with the previous generation, after $1 \mathrm{wk}$ the mated pair was removed and placed in a new container with new bolts of EAB-infested tropical ash. This was repeated weekly until the female Spathius died. Once emergence had completed, bolts were dissected, the fate of each EAB larvae was scored, and any unemerged parasitoids were counted (as described above).

\section{Field Sampling of EAB Larvae}

To determine EAB larval life stage in the field locally, two sites were located in Virginia, at Douthat State Park, Millboro, VA (37.89409,
-79.8015) and Mid-County Park, Christiansburg, VA (37.17123, -80.4119; MCP). Each site was visited once per month during the summer of 2017. One to two symptomatic trees were selected, felled, and debarked in 1-m sections using methods described in USDAAPHIS/ARS/FS (2019). All EAB larvae present were collected and their lifestage was assessed. All larvae were brought back to the PFRC and observed for $3 \mathrm{wk}$ for any endoparasitoid emergence.

\section{Statistical Analyses}

The nonparametric Kaplan-Meier survival platform (Kaplan and Meier 1958) was used to calculate the median emergence time and $95 \%$ confidence intervals for all four cohorts. Days since exposure to Virginia field conditions was used as the time to event, and the count of daily emergence used as the frequency of the event. This was performed following the protocol outlined in Duan et al. (2011). A log-rank and Cox proportional hazard test (based on the KaplanMeier survival platform) were performed to determine significance in differences between pairs of treatments ( $S$. agrili cold-treated and untreated S. agrili, S. galinae cold-treated and untreated S. galinae, $S$. agrili cold-treated and $S$. galinae cold-treated, untreated $S$. agrili and untreated $S$. galinae). If no difference was found between cold acclimatization treatment and untreated, then treatment groups were pooled for comparisons between $S$. agrili and $S$. galinae. Due to multiple comparisons across all groups of cohorts, then four pairwise comparisons, the Bonferroni correction was used and the type I error rate (alpha) was set to 0.1 .

Longevity of adult parasitoids was calculated in weeks using a one-way ANOVA to determine differences between treatments within each species, and between species. Due to a low number of individuals followed through their lifetime, cohorts were pooled by species, with the assumption that cold acclimatization treatment had no effect on longevity. Sex ratio was determined by calculating the proportion of female to male progeny, any replicates with only male offspring were removed from the analysis due to haplodiploidy. The weekly replicates were used to determine parasitism rate by dividing the number of parasitized larvae by the total larvae provided, and fecundity based on a count of the parasitoid progeny per individual female. Data were then analyzed in a one-way ANOVA to determine differences between cold acclimatization treatment and untreated within species.

For assessment of the subsequent generation, the Kaplan-Meier survival platform was used again to determine differences between pairs of cohort treatments, as was performed for the previous generation emergence. Median emergence time and 95\% confidence intervals were calculated through the survival platform. Log-rank and Cox proportional hazard test were performed between pairs to determine significance. Multigenerational effects on parasitism rate were assessed using a count of EAB larvae fate analyzed by a oneway ANOVA. Again, due to multiple comparisons across all groups of cohorts, then four pairwise comparisons, the Bonferroni correction was used and the type I error rate (alpha) was set to 0.1 for emergence analysis.

All data were analyzed in SAS JMP Pro 14.0.0 (SAS Institute 2019).

\section{Results}

\section{Early Season Emergence}

Emergence of cold acclimatized S. agrili began on 2 May and completed emergence on 14 June. Untreated S. agrili emergence began on 5 May and completed emergence on 16 June. Emergence of both 
Table 1. The summary of the Kaplan-Meier survival curve for early season emergence of S. agrili and S. galinae under cold acclimatization and untreated conditions

\begin{tabular}{lccccccc}
\hline Group & $\begin{array}{c}\text { Adults } \\
\text { emerged }\end{array}$ & $\begin{array}{c}\text { Median } \\
\text { Time (d) }\end{array}$ & $\begin{array}{c}\text { Median Time } \\
\text { (calendar date) }\end{array}$ & Lower 95\% & Upper 95\% & 25\% Emerged & 75\% Emerged \\
\hline Cold Acclimatized S. agrili & 30 & $79.5 \mathrm{a}$ & May 26 & 76 & 82 & 70 & 82 \\
Cold Acclimatized S. galinae & 85 & $93 \mathrm{c}$ & June 9 & 83 & 95 & 78 & 78 \\
Untreated S. agrili & 48 & $82.5 \mathrm{a}$ & May 29 & 82 & 83 & 78.5 & 79 \\
Untreated S. galinae & 381 & $68 \mathrm{~b}$ & May 15 & 68 & 70 & & \\
Combined & 544 & & & & & & \\
\hline
\end{tabular}

a. Significant differences in median emergence time are indicated by lettered grouping next to median time. Comparison between all groups indicated significant differences between groups. Pairwise analysis between $S$. galinae treatments indicated cold acclimatization significantly delayed emergence. Pairwise analysis between untreated S. agrili and untreated S. galinae indicated S. agrili untreated emerged significantly later than S. galinae untreated. Pairwise analysis between cold acclimatized S. agrili and cold acclimatized S. galinae indicated that cold acclimatized S. agrili emerged significantly later than cold acclimatized S. galinae. Pairwise analysis between $S$. agrili treatments indicated no difference.

b. Groups without a factor (species or treatment) were not compared.

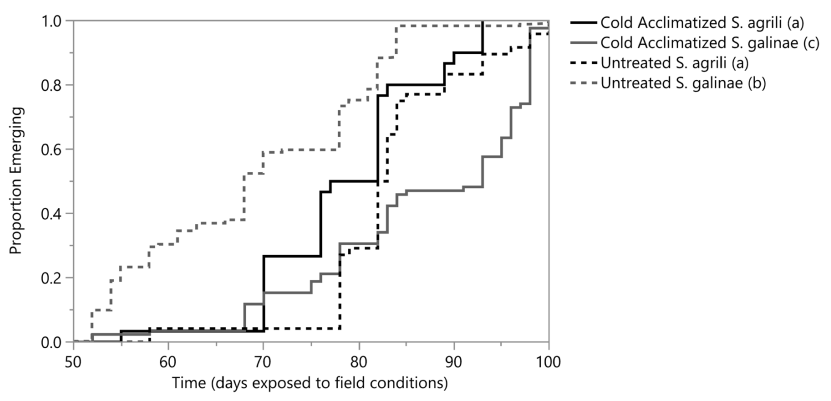

Fig. 2. Daily emergence of $S$. agrili (shown in black) and $S$. galinae (shown in gray) adults over time. Cold acclimatization and untreated control groups. Significant difference from pairwise comparisons is indicated by letter grouping in the legend (Log-Rank, $P<0.01$ ).

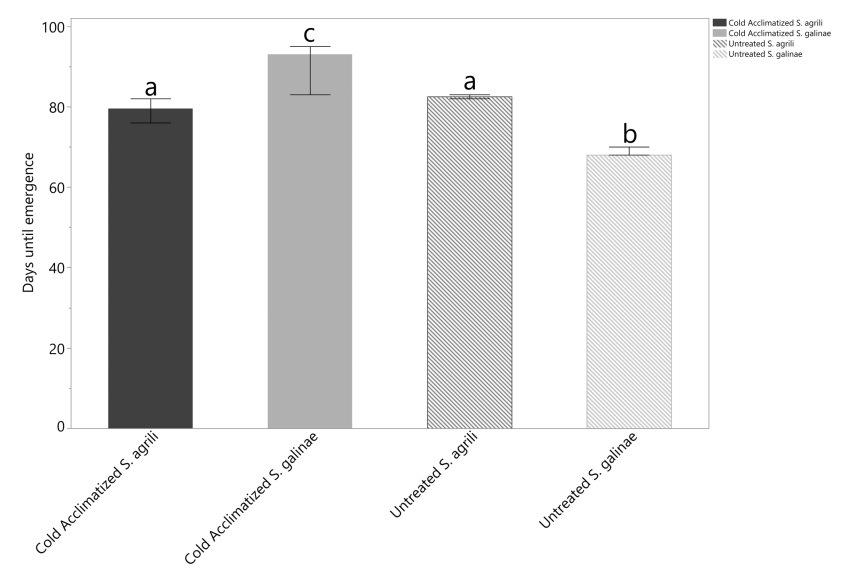

Fig. 3. Median days to emergence for both cohorts of S. agrili and S. galinae $(95 \% \mathrm{Cl}$ for the Kaplan-Meier survival curve). Significant difference is indicated by letter grouping (Log-Rank, $P<0.01$ ).

cohorts of S. galinae began on 29 April and lasted until 16 June when emergence ended (Table 1 and Fig. 2). There were significant differences in emergence times among the four cohorts (Log-Rank $\chi^{2}=128.038, \mathrm{df}=3, P<0.0001$; Table 1 and Fig. 2). Pairwise comparisons showed that cold acclimatization treatment significantly delayed the emergence time of $S$. galinae compared with untreated S. galinae (Log-Rank $\chi^{2}=91.856, \mathrm{df}=1, P<0.0001$; Table 1 and Fig. 3). Emergence of untreated $S$. agrili was significantly later than untreated S. galinae (Log-Rank $\chi^{2}=36.193, \mathrm{df}=1, P<0.0001$;
Table 1 and Fig. 3). In contrast, cold acclimatized S. agrili emerged earlier than cold acclimatized S. galinae (Log-Rank $\chi^{2}=20.723$, $\mathrm{df}=1, P<0.0001$; Table 1 and Fig. 3 ). Cold acclimatization treatment did not affect emergence time of $S$. agrili compared with untreated $S$. agrili $\left(\log -\operatorname{Rank} \chi^{2}=5.01, \mathrm{df}=1, P=0.0252\right.$; Table 1 and Fig. 3).

\section{Early Season Longevity, Parasitism Rate, Sex Ratio, and Fecundity}

Both $S$. agrili and $S$. galinae cohorts were pooled regardless of treatment due to limited replicates. In total, 21 pairs of $S$. galinae were set up, whereas a total of 10 pairs of $S$. agrili were set up. Longevity did not differ between S. agrili and S. galinae (Table 2). Cold acclimatization treatment did not affect parasitism rate for either S. agrili or S. galinae (Table 2). Due to lack of differences among treatments, all treatments were pooled by species and no difference was detected between parasitism rate of $S$. agrili and S. galinae (Table 2). Cold acclimatization treatment did not affect sex ratio for either $S$. agrili or S. galinae (Table 2). When groups were pooled regardless of treatment, sex ratio of $S$. agrili did not differ from $S$. galinae. It also did not affect fecundity for either $S$. agrili or $S$. galinae (Table 2). When groups were pooled regardless of treatment, fecundity of $S$. agrili was significantly greater than S. galinae (Table 2).

\section{Subsequent Generation Emergence}

There was an overall significant difference in subsequent generation emergence time among the four cohorts (Log-Rank $\chi^{2}=92.9856$, $\mathrm{df}=3, P<0.0001$; Table 3 and Fig. 4). Pairwise comparisons showed that the median emergence time of cold acclimatized S. agrili progeny was significantly longer than that of untreated $S$. agrili progeny $\left(\right.$ Log-Rank $\chi^{2}=15.5906, \mathrm{df}=1, P<0.0001$; Table 3 and Fig. 5$)$. In contrast, there was no significant difference in emergence timing between cold acclimatized $S$. galinae and untreated S. galinae (LogRank $\chi^{2}=1.30, \mathrm{df}=1, P=0.254$; Table 3 and Fig. 5).

Furthermore, there were significant differences in emergence time between the two parasitoid species. Cold acclimatized S. agrili had peak emergence significantly earlier than cold acclimatized $S$. galinae (Log-Rank $\chi^{2}=10.88, \mathrm{df}=1, P=0.0010$; Table 3 and Fig. 5). Untreated $S$. galinae progeny began emergence earlier than untreated $S$. agrili progeny, although both completed emergence in similar lengths of time $\left(\log -\operatorname{Rank} \chi^{2}=17.84, \mathrm{df}=1, P<0.0001\right.$; Table 3 and Fig. 5). 
Table 2. The longevity, parasitism rate, sex ratio, and fecundity of the early season emergence of all four cohorts by treatment and pooled by species where no difference occurred

\begin{tabular}{lcccc}
\hline Cohort & ${\text { Longevity }(w k)^{\mathrm{a}}}$ & Parasitism Rate $\left(\%\right.$ attacked) $^{\mathrm{b}}$ & Sex Ratio $\left(\%\right.$ Female Progeny) $^{\mathrm{c}}$ & Fecundity (Progeny/Adult) $^{\mathrm{d}}$ \\
\hline Cold acclimatized S. agrili & - & $35.67 \pm 7.30$ & $59.94 \pm 14.26$ & $35.00 \pm 14.97$ \\
Untreated S. agrili & - & $28.48 \pm 5.32$ & $60.54 \pm 12.75$ & $33.00 \pm 10.59$ \\
Cold acclimatized S. galinae & - & $26.56 \pm 4.64$ & $59.51 \pm 8.39$ & $19.00 \pm 3.99$ \\
Untreated S. galinae & - & $20.86 \pm 6.25$ & $49.07 \pm 12.51$ & $14.40 \pm 5.35$ \\
S. agrili (pooled) & $5.22 \pm 0.79$ & $30.97 \pm 4.05$ & $60.28 \pm 8.94$ & $33.67 \pm 5.87$ \\
S. galinae (pooled) & $4.40 \pm 0.61$ & $24.54 \pm 3.94$ & $56.27 \pm 7.04$ & $17.35 \pm 4.70$ \\
\hline
\end{tabular}

Pairwise analyses were only performed selectively based on biologically significant comparisons (ANOVA $P<0.05$ ).

a Pairwise analysis indicated no significant difference $(F=0.6793 ; \mathrm{df}=1,23 ; P=0.4187)$.

${ }^{b}$ Pairwise analysis between Cold acclimatized $S$. agrili and untreated indicated no difference $(F=0.6343 ; \mathrm{df}=1,71 ; P=0.4285)$. Pairwise analysis between Cold acclimatized $S$. galinae and untreated indicated no difference $(F=0.5362 ; \mathrm{df}=1,75 ; P=0.4663)$. Pairwise analysis between $S$. agrili (pooled) and $S$. galinae (pooled) indicated no difference $(F=1.2940 ; \mathrm{df}=1,147 ; P=0.2572)$.

'Pairwise analysis between cold acclimatized $S$. agrili and untreated indicated no difference $(F=0.0010 ; \mathrm{df}=1,17 ; P=0.9749)$. Pairwise analysis between cold acclimatized $S$. galinae and untreated indicated no difference $(F=0.4802 ; \mathrm{df}=1,28 ; P=0.4943)$. Pairwise analysis between $S$. agrili (pooled) and $S$. galinae (pooled) indicated no difference $(F=0.1238 ; \mathrm{df}=1,45 ; P=0.7266)$.

${ }^{\mathrm{d}}$ Pairwise analysis between cold acclimatized $S$. agrili and untreated indicated no difference $(F=0.0119 ; \mathrm{df}=1,8 ; P=0.9162)$. Pairwise analysis between cold acclimatized $S$. galinae and untreated indicated no difference $(F=0.4758 ; \mathrm{df}=1,13 ; P=0.5034)$. Pairwise analysis between $S$. agrili (pooled) and $S$. galinae (pooled) indicated significant difference $(F=4.7071 ; \mathrm{df}=1,22 ; P=0.0417)$.

Table 3. Subsequent generation (progeny) emergence of Spathius agrili and Spathius galinae from parents under cold acclimatization and untreated conditions from the Kaplan-Meier survival curve

\begin{tabular}{|c|c|c|c|c|c|c|}
\hline Group & Adults Emerged & Median Time (d) & Lower $95 \%$ & Upper $95 \%$ & $25 \%$ Emerged & $75 \%$ Emerged \\
\hline Cold Acclimatized S. agrili & 68 & $38 \mathrm{a}$ & 37 & 43 & 36.5 & 53.5 \\
\hline Cold Acclimatized S. galinae & 118 & $45 \mathrm{~b}$ & 44 & 47 & 42 & 59 \\
\hline Untreated $S$. agrili & 75 & $36 \mathrm{c}$ & 36 & 39 & 35 & 40 \\
\hline Untreated S. galinae & 31 & $45 \mathrm{~b}$ & 45 & 48 & 43 & 50 \\
\hline Combined & 292 & & & & & \\
\hline
\end{tabular}

a. Significant differences in median emergence time are indicated by lettered grouping next to median time (Log-Rank, $P<0.01$ ).

b. Groups without a factor (species or treatment) were not compared.



Fig. 4. Daily emergence of all subsequent generation cohorts. Spathius agrili (shown in black), S. galinae (shown in gray), cold-treated (shown in solid lines), and untreated (shown in dashed lines). Significant difference is indicated by letter grouping in the legend, (Log-Rank, $P<0.01$ ).

\section{Subsequent Generation Parasitism Rate}

There was no significant multigenerational effect of cold acclimatization treatment on the lifelong parasitism rate of the subsequent generation for either $S$. agrili $(F=0.1935, \mathrm{df}=1.74, P=0.6613)$ or $S$. galinae $(F=0.0063, \mathrm{df}=1.56, P=0.9368)$. When groups were pooled regardless of treatment parasitism rates did not differ between species $(F=0.4394, \mathrm{df}=1.131, P=0.5086$; Table 4).

\section{EAB Larval Phenology in the Field}

Very few EAB larvae were available in June, and at Douthat State Park. The majority of EAB sampled were still pupae. Total EAB larvae increased in July, with third- and fourth-instar larvae increasing to

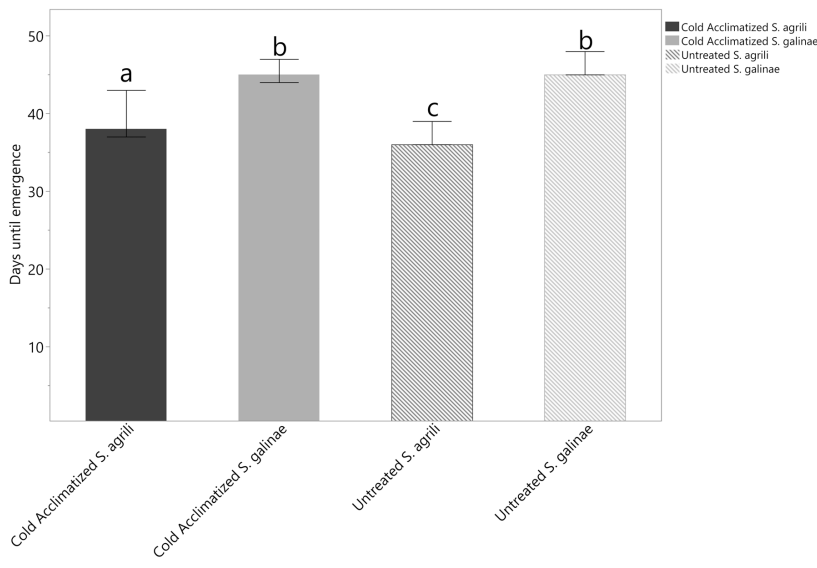

Fig. 5. Median days to emergence for the subsequent generation of both cohorts of S. agrili and S. galinae $(95 \% \mathrm{Cl}$ for the Kaplan-Meier survival curve). Significant difference is indicated by letter grouping (Log-Rank, $P<0.01)$.

their maximum abundance. In August, the number of EAB larvae declined although some larvae were still present (Fig. 6). At MCP, a similar pattern was observed, but with much lower overall numbers.

\section{Discussion}

Early season emergence of $S$. agrili and $S$. galinae differed under Virginia field conditions. The two species displayed differential 
responses to cold acclimatization. Median emergence of untreated S. galinae occurred on May 15, approximately $2 \mathrm{wk}$ (11.5-14.5 d) earlier than S. agrili, and $25 \mathrm{~d}$ earlier than cold acclimatized S. galinae. The emergence time of $S$. agrili was unaffected by the cold acclimatization period, median emergence occurred on May 26 (cold acclimatized) and May 29 (untreated). The last cohort to emerge was cold acclimatized $S$. galinae, median emergence occurred on June 9. With an average of 4 to 5 wk lifespan, both $S$. agrili and S. galinae, regardless of treatment, overlapped with third- and fourth-instar $\mathrm{EAB}$ at both sampled field sites. Under our experimental conditions, $S$. agrili and cold acclimatized $S$. galinae emerged later in the season when more third- and fourth-instar EAB are likely to be found in field conditions. Untreated $S$. galinae had very little overlap with available EAB larvae due to their earlier peak emergence.

Both S. agrili and S. galinae had shorter adult longevity than literature suggested. Both species of Spathius have similar adult longevity, 4.40-5.22 $\mathrm{wk}$ in this experiment, lower than their 7-8 wk longevity under laboratory rearing conditions (Gould et al. 2011, Duan et al. 2014). This could be due to differences in testing methodologies, or an effect of Virginia climate conditions.

Table 4. The parasitism rate of the subsequent generation of S. agrili and S. galinae

\begin{tabular}{lc}
\hline Cohort & Parasitism Rate $(\%$ attacked $)$ \\
\hline S. agrili (pooled) & $23.52 \pm 3.67$ \\
Cold acclimatized S. agrili & $22.01 \pm 4.97$ \\
Untreated S. agrili & $25.16 \pm 5.17$ \\
S. galinae (pooled) & $27.23 \pm 4.21$ \\
Cold acclimatized S. galinae & $26.66 \pm 8.35$ \\
Untreated S. galinae & $27.45 \pm 5.22$
\end{tabular}

No groups differed when compared using pairwise ANOVA ( $P$ o groups
Cold acclimatization had multigenerational effects on emergence time of $S$. agrili, but not on $S$. galinae. The subsequent generation of $S$. agrili emerged $2 \mathrm{~d}$ earlier when the parent generation was cold acclimatized. Emergence of $S$. galinae progeny was unaffected by cold acclimatization. Cold acclimatization treatment did not affect longevity, sex ratio of progeny, or parasitism rate in either species. Differences were observed between the two species when cold acclimatized and untreated cohorts were pooled within species. The fecundity of $S$. galinae was lower than S. agrili which is consistent with previous literature of the both species (Gould et al. 2011, Belokobylskij et al. 2012, Duan et al. 2014, Watt et al. 2016).

Depending on the availability of EAB larvae at a given site, a cold treatment could be used to improve phenological synchronization between the phenology of the parasitoids and their host. With a better understanding of EAB phenology at individual sites, this early season release method could allow for infested bolts to be shipped to field sites earlier in the season and aid in mass rearing. In the time since this experiment was completed, further climate matching has been completed on $S$. agrili and $S$. galinae, based on EAB overwintering lifestage across its invasive range (USDA-APHIS/ARS/FS 2019). The USDA now suggests release of $S$. agrili only south of the 40th parallel due to its failure to establish in the northern United States, and $S$. galinae only north of it due to early spring larval availability (USDA-APHIS/ARS/FS 2019). As S. galinae establishes in the northern United States and Canada where $S$. agrili did not, each will contribute to the control of EAB as part of a complex of biological control agents in different geographical regions (Larson and Duan 2016, Duan et al. 2019). As EAB biological control guidelines are updated with new information on EAB larval development, parasitoid release timing is becoming more precise, and less likely to miss the critical period of larval availability. Therefore, this method of early season release would not be suitable for $S$. galinae without a cold treatment. Spathius agrili could be released this way, because

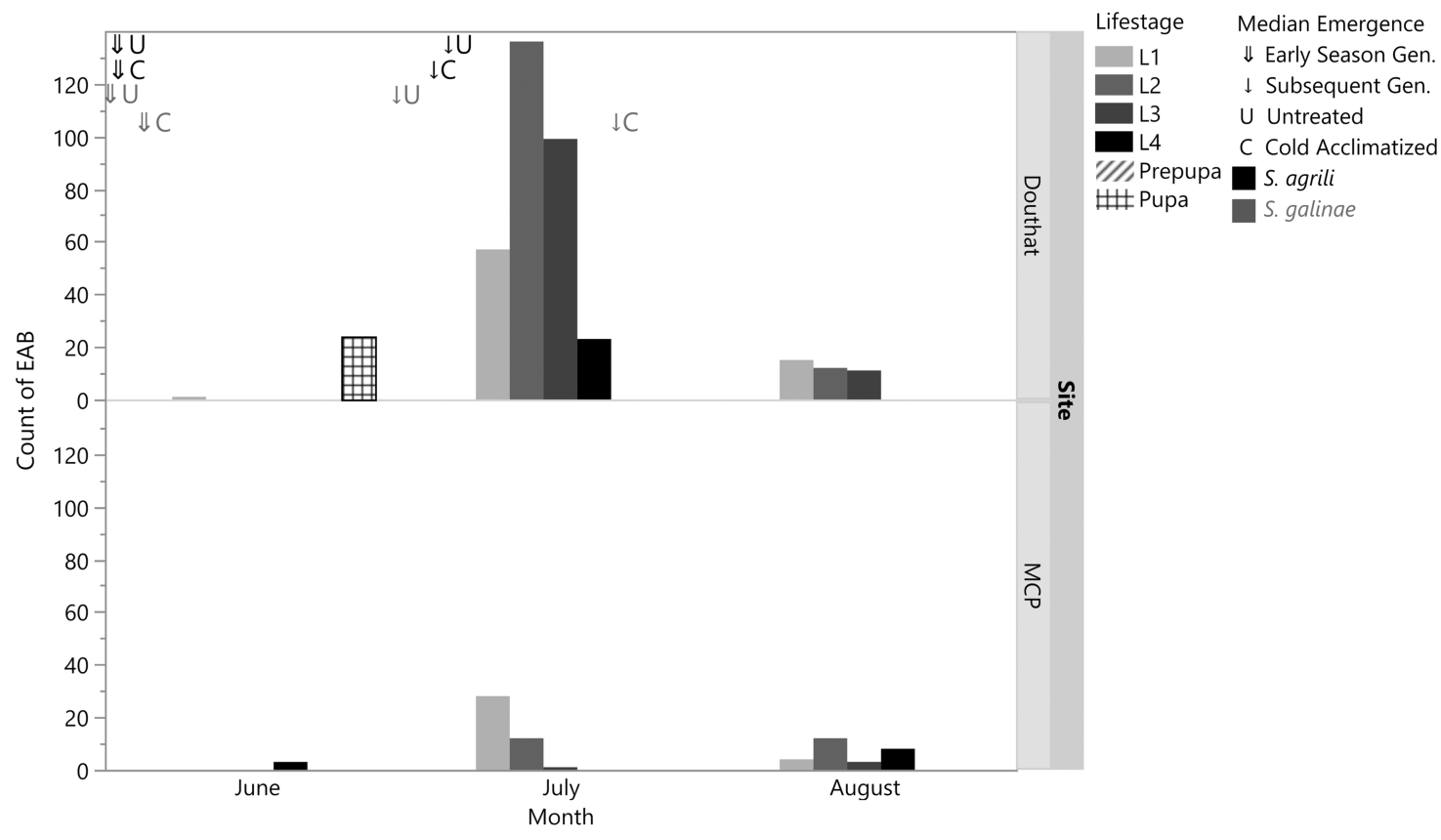

Fig. 6. Sum of all EAB larvae sorted by lifestage from monthly sampling at each field site. Sampling at Douthat took place on 15 June 2017 , 13 July 2017 , and 16 August 2017. Sampling at MCP took place on 2 June 2017, 11 July 2017, and 14 August 2017. Median parasitoid emergence date are indicated above EAB larval count. Subsequent generation placement is based on the assumption that the parent generation emerges at the median emergence time, and there is no preoviposition period of the parent generation. Placement is an approximation and is not to scale. 
emergence occurred when third- and fourth-instar EAB were present at both sites regardless of treatment.

Further studies could examine these patterns using true overwintering conditions, or in whole tree scenarios. Whole tree sampling in field scenarios should give the true representation of the overwintering survival and early season emergence of both species, and should be conducted in the future. Additional work should focus on longer cold exposures to simulate natural winters, a more varied temperature regime to observe impacts across a range of climate change conditions, as well as geographical ranges, and larger sample sizes if possible.

\section{Acknowledgments}

We would like to thank Brandi Benedict, Fitz Cherry, and Rachel Campbell for the hours of bolt dissections, emergence counting, and colony maintenance. Thank you to Kristi Larson, the BIIRU lab, and the APHIS Brighton Facility for rearing the insects for these experiments. Additional thanks to Erin Langton-Ragozzino, Miranda Pacheco, and Liam Sullivan for graciously helping review an early draft of this manuscript. This was funded through a U.S. Forest Service Cooperative Forest Health Grant 15-DG-11083150-042 and USDA ARS Grant 58-8010-5-016.

\section{References Cited}

Bauer, L. S., H. Liu. 2007. Oobius Agrili (Hymentoptera: Encyrtidae), a solitary egg parasitoid of emerald ash borer from China, pp. 63-64. In Mastro, Victor; Lance, David; Reardon, Richard; Parra, Gregory, comps. Emerald ash borer and Asian longhorhed beetle research and development review meeting; 2006 October 29-November 2; Cincinnatti, OH. FHTET 2007-04. U.S. Forest Service, Forest Health Technology Enterprise Team, Morgantown, WV.

Bauer, L. S., H. Liu, D. Miller, and J. Gould. 2008. Developing a classical biological control program for Agrilus planipennis (Coleoptera: Buprestidae), an invasive ash pest in North America. Newsl. Michigan Entomol. Soc. 53: $38-39$.

Belokobylskij, S. A., G. I. Yurchenko, J. S. Strazanac, A. Zaldívar-riverón, and V. Mastro. 2012. A new emerald ash borer (Coleoptera : Buprestidae) Parasitoid Species of Spathius Nees (Hymenoptera: Braconidae: Doryctinae) from the russian far East and South Korea. Ann. Entomol. Soc. Am. 105(2): 165-178.

Bray, A. M., L. S. Bauer, T. M. Poland, R. A. Haack, A. I. Cognato, and J. J. Smith. 2011. Genetic analysis of emerald ash borer (Agrilus planipennis Fairmaire) populations in Asia and North America. Biol. Invasions. 13: 2869-2887.

Canadian Food Inspection Agency. 2019. Areas regulated for the emerald ash borer. https://inspection.gc.ca/plants/plant-pests-invasive-species/insects/ emerald-ash-borer/eng/1337273882117/1337273975030 (accessed 7 August 2019).

Cappaert, D., D. G. Mccullough, T. M. Poland, and N. W. Siegert. 2005. Emerald ash borer in North America a research and regulatory challenge. Am. Entomol. 51: 152-165.

Davidson, W., and L. K. Rieske. 2016. Establishment of classical biological control targeting emerald ash borer is facilitated by use of insecticides, with little effect on native arthropod communities. Biol. Control. 101: $78-86$.

Duan, J. J., C. B. Oppel, M. D. Ulyshen, L. S. Bauer, and J. Lelito. 2011. Biology and Life History of Tetrastichus planipennisi (Hymenoptera: Eulophidae), a Larval Endoparasitoid of the Emerald Ash Borer (Coleoptera: Buprestidae). Florida Entomol. 94: 933-940.

Duan, J. J., L. S. Bauer, K. J. Abell, and R. van Driesche. 2012. Population responses of hymenopteran parasitoids to the emerald ash borer (Coleoptera: Buprestidae) in recently invaded areas in north central United States. BioControl. 57: 199-209.

Duan, J. J., T. J. Watt, and K. Larson. 2014. Biology, life history, and laboratory rearing of Spathius galinae (Hymenoptera: Braconidae), a larval parasitoid of the invasive emerald ash borer (Coleoptera: Buprestidae). J. Econ. Entomol. 107: 1-8.

Duan, J. J., L. S. Bauer, K. J. Abell, M. D. Ulyshen, and R. G. Van Driesche. 2015. Population dynamics of an invasive forest insect and associated natural enemies in the aftermath of invasion: implications for biological control. J. Appl. Ecol. 52: 1246-1254.

Duan, J. J., L. S. Bauer, R. G. van Driesche, and J. R. Gould. 2018. Progress and challenges of protecting North American ash trees from the emerald ash borer using biological control. Forests. 9: 1-17.

Duan, J. J., R. G. Van Driesche, R. S. Crandall, J. M. Schmude, C. E. Rutledge, B. H. Slager, J. R. Gould, and J. S. Elkinton. 2019. Establishment and early impact of Spathius galinae (Hymenoptera: Braconidae) on Emerald ash borer (Coleoptera: Buprestidae) in the Northeastern United States. J. Econ. Entomol. 112: 2121-2130.

Emerald Ash Borer Information. 2019. Emerald ash borer information. Emerald ash borer information network. Available online: http://www. emeraldashborer.info/ (accessed on 28 July 2019).

Federal Register. 2015. Availability of an environmental assessment for field release of the parasitoid Spathius galinae for the biological con- trol of the emerald ash borer (Agrilus planipennis) in the contiguous United States. Fed. Regist. 80: 7827-7828, [docket number APHIS-20140094]. Available online: https://www.regulations.gov/docket?D=APHIS-2014-0094 (accessed on 15 June 2018).

Gould, J. R., T. Ayer, and I. Fraser. 2011. Effects of rearing conditions on reproduction of Spathius agrili (Hymenoptera: Braconidae), a parasitoid of the emerald ash borer (Coleoptera: Buprestidae). J. Econ. Entomol. 104: 379-387.

Haack, R. A., E. Jendek, H. Liu, K. R. Marchant, T. R. Petrice, and T. M. Poland. 2002. The emerald ash borer: a new exotic pest in North America. Newsl. Michigan Entomol. Soc. 47: 1-5.

Hooie, N. A., G. J. Wiggins, P. L. Lambdin, J. F. Grant, S. D. Powell, and J. P. Lelito. 2015. Native parasitoids and recovery of Spathius agrili from areas of release against emerald ash borer in eastern Tennessee, USA. Biocontrol Sci. Technol. 25: 345-351.

Jennings, D. E., J. J. Duan, D. Bean, J. R. Gould, K. A. Rice, and P. M. Shrewsbury. 2016. Monitoring the establishment and abundance of introduced parasitoids of emerald ash borer larvae in Maryland, U.S.A. Biol. Control. 101: 138-144.

Kaplan, E. L., Meier, P. 1958. Nonparametric estimation from incomplete observations. J. Am. Stat. Assoc. 53: 457-481.

Larson, K. M., and J. J. Duan. 2016. Differences in the reproductive biology and diapause of two Congeneric species of egg parasitoids (Hymenoptera : Encyrtidae) from northeast Asia : implications for biological control of the invasive emerald ash borer (Coleoptera : Buprestidae). Biol. Control. 103: 39-45.

Liu, H., L. S. Bauer, D. L. Miller, T. Zhao, R. Gao, L. Song, Q. Luan, R. Jin, and C. Gao. 2007. Seasonal abundance of Agrilus planipennis (Coleoptera: Buprestidae) and its natural enemies Oobius agrili (Hymenoptera: Encyrtidae) and Tetrastichus planipennisi (Hymenoptera: Eulophidae) in China. Biol. Control. 42: 61-71.

MapBioControl. 2019. Agent release tracking and data management for federal, state, and researchers releasing three biocontrol agents released against emerald ash borer. http://www.mapbiocontrol.org/ (accessed 7 August 2019).

Mercader, R. J., D. G. McCullough, A. J. Storer, J. M. Bedford, R. Heyd, T. M. Poland, and S. Katovich. 2015. Evaluation of the potential use of a systemic insecticide and girdled trees in area wide management of the emerald ash borer. For. Ecol. Manage. 350: $70-80$.

SAS Institute Inc. 2019. JMP Pro 14.0.0. SAS Institute Inc. SAS Campus Drive, Gary, NC.

Siegert, N. W., D. G. Mccullough, A. M. Liebhold, and F. W. Telewski. 2014. Dendrochronological reconstruction of the epicentre and early spread of emerald ash borer in North America. J. Conserv. Biogeogr. 847-858.

USDA-APHIS/ARS/FS. 2019. Emerald ash borer biological control release and recovery guidelines. USDA-APHIS-ARS-FS, Riverdale, MD. https://www.aphis.usda.gov/plant_health/plant_pest_info/ emerald_ash_b/downloads/EAB-FieldRelease-Guidelines.pdf (accessed September 30, 2019). 
Watt, T. J., and J. J. Duan. 2014. Influence of host age on critical fitness parameters of Spathius galinae (Hymenoptera: Braconidae), a new parasitoid of the emerald ash borer (Coleoptera: Buprestidae). J. Econ. Entomol. 107: 1320-1329.

Watt, T. J., J. J. Duan, D. W. Tallamy, J. Hough-Goldstein, and T. W. Ilvento. 2016. Reproductive and developmental biology of the emerald ash borer parasitoid Spathius galinae (Hymenoptera: Braconidae) as affected by temperature. Biol. Control. 96: 1-7.

Yang, Z., J. S. Strazanac, P. M. Marsh, C. Van Achterberg, and W. Choi. 2005. First recorded parasitoid from China of Agrilus planipennis: a new species of Spathius (Hymenoptera: Braconidae: Doryctinae). Ann. Entomol. Soc. Am. 98: 636-642.
Yang, Z., J. S. Strazanac, Y.-X. Yao, and X. Wang. 2006. A new species of emerald ash borer parasitoid from China belonging to the genus Tetrastichus Haliday (Hymenoptera: Eulophidae). Proc. Entomol. Soc. Washingt. 108: 550-558.

Yang, Z. Q., X. Y. Wang, J. R. Gould, R. C. Reardon, Y. N. Zhang, G. J. Liu, and E. S. Liu. 2010. Biology and behavior of Spathius agrili, a parasitoid of the emerald ash borer, Agrilus planipennis, in China. J. Insect Sci. 10: 30.

Zhang, Y. Z., D. W. Huang, T. H. Zhao, H. P. Liu, and L. S. Bauer. 2005. Two new species of egg parasitoids (Hymenoptera: Encyrtidae) of wood-boring beetle pests from China. Phytoparasitica. 33: 253-260. 